\title{
Optical Bragg Gratings in Inorganic-Organic Hybrid Polymers for Highly Sensitive Temperature Measurements
}

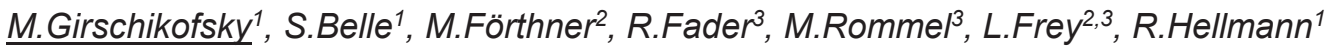 \\ 1 University of Applied Sciences Aschaffenburg, Würzburger Straße 45, \\ 63743 Aschaffenburg, Germany, maiko.girschikofsky@h-ab.de \\ ${ }^{2}$ Chair of Electron Devices, Friedrich Alexander University Erlangen-Nuremberg, \\ Cauerstraße 6, 91058 Erlangen, Germany \\ ${ }^{3}$ Fraunhofer Institute for Integrated Systems and Device Technology (IISB), \\ Schottkystraße 10, 91058 Erlangen, Germany
}

\begin{abstract}
:
We report on the fabrication of an optical sensor based on channel waveguide Bragg gratings in precured inorganic-organic ORMOCER ${ }^{\circledR}$ hybrid-polymer substrates. The particular combination of the optical component and chosen substrate lead to a planar structure with ideal characteristics for highly sensitive temperature measurements. The resulting sensor element shows a linear dependence between temperature and sensor signal with a markedly high sensitivity of up to $294 \mathrm{pm} \mathrm{K}^{-1}$, a thirtyfold higher sensitivity as compared to commonly used silica based fiber Bragg gratings. For the applied interrogation system this sensitivity corresponds to a notable resolution of $20 \mathrm{mK}$ for temperature recordings.
\end{abstract}

Key words: Bragg Grating, Temperature Sensor, Inorganic-Organic Hybrid Polymer, ORMOCER ${ }^{\circledR}$

\section{Introduction}

Due to numerous advantages like immunity to electromagnetic fields or long distance remote controllability fiber-optic sensor devices have become a common technology in the field of temperature sensing. An ever frequently discussed type of fiber-optic temperature sensors are Fiber Bragg Grating (FBG) temperature sensors [1-2]. This type of sensor is based on a periodic modulation of the refractive index within the fiber core causing a defined portion of the in-coupled optical spectrum to be reflected, whereat the reflected wavelength $\lambda_{B}$ depends on the effective refractive index $n_{\text {eff }}$ and the gratings modulation period $\Lambda$ as given in equation (1).

$$
\lambda_{B}=2 n_{\text {eff }} \wedge
$$

The applicability of Bragg gratings (BG) in the field of temperature sensing is favored by the dependence of both, the refractive index and the Bragg grating period on the temperature, where the shift of the reflected wavelength due to temperature changes can be expressed as shown in equation (2).

$$
\Delta \lambda_{B}=\lambda_{0}(\alpha+\zeta) \Delta T
$$

Where $\alpha=(\partial \Lambda / \partial T) \Lambda^{-1}$ represents the coefficient of thermal expansion (CTE) and $\zeta=(\partial n / \partial T) n^{-1}$ the thermo-optic coefficient (TOC), which both are material-specific parameters.

Usually, Bragg gratings are fabricated in silica based optical fibers but polymer waveguides are as well under investigation to host Bragg gratings for sensing applications [1-3]. The characteristics of these materials, such as the CTE and the TOC, lead to sensitivities of $11 \mathrm{pm} / \mathrm{K}$ for silica $[1,2]$ and $-55 \mathrm{pm} / \mathrm{K}$ to $-100 \mathrm{pm} / \mathrm{K}$ for polymer based waveguide Bragg gratings [2,4]. Accordingly, polymers represent the more suitable material for hosting Bragg gratings when considering higher temperature sensitivities [5]. However, as compared to silica based Bragg gratings the operable temperature range of common polymer Bragg gratings (PBG) is generally lower as being limited by the glass transition temperature of the polymer [5]. In addition, most PBGs are not qualified in the presence of solvents which may dissolve the polymer. A more recent class of polymers that bridges the gap between high sensitivity and high chemical and thermal stability are inorganic-organic hybrid polymers such as the organically modified ceramics ORMOCER ${ }^{\circledR}[6]$. This particular class of material is widely studied for the fabrication of wafer scale optical elements [7] and is due to its beneficial material properties $[8,9]$ already under consideration as an additive applied to common silica 
based fiber Bragg gratings in order to enhance their temperature sensitivity [10-12]. In this contribution, we therefore discuss the fabrication of volumetric planar waveguide Bragg gratings for temperature measurements in pre-cured inorganic-organic ORMOCER ${ }^{\circledR}$ hybrid-polymers.

\section{Experimental}

Different commercially available ORMOCER ${ }^{\circledR} \mathrm{S}$, which have been specifically designed for the fabrication of optical elements, namely OrmoCore $^{\circledR}$, OrmoClad ${ }^{\circledR}$ and OrmoComp ${ }^{\circledR}$ (micro resist technology), have been employed as purchased in this study. For the fabrication of the planar hybrid-polymer substrates, the ORMOCER ${ }^{\circledR} S$ are spin coated onto an oxygen plasma pre-treated silicon wafer and subsequently cured by UV irradiation using a mercuryvapor lamp at an exposure dose of $2.5 \mathrm{~J} / \mathrm{cm}^{2}$ (quantified for the i-line) resulting in a homogenous resist layer thickness of about $14 \mu \mathrm{m}$. Before and after UV irradiation the hybrid-polymer substrates are applied to a 2 minute pre-exposure bake at $80^{\circ} \mathrm{C}$ and a 10 minute post-exposure bake at $130^{\circ} \mathrm{C}$, respectively. In case of OrmoCore ${ }^{\circledR}$ and OrmoClad $^{\circledR}$, an inhibition layer (a small layer of uncured material) can be found af- ter the UV irradiation, which necessitates an additional development step and a subsequent 10 minute hardbake at $130^{\circ} \mathrm{C}$. For the fabrication of waveguides, a KrF excimer laser (Bragg Star Industrial by Coherent) is used operating at a wavelength of $\lambda=248 \mathrm{~nm}$, a repetition rate of $200 \mathrm{~Hz}$ and a pulse energy of $E_{p}=12 \mathrm{~mJ}$. The waveguide inscription is carried out with the laser beam homogenously expanded using a fly's eye condenser (SÜSS MicroTec) and a chromium amplitude mask in soft contact to the pre-cured hybrid-polymer substrate at a laser fluence of $15 \mathrm{~J} / \mathrm{cm}^{2}$. By this measure, 6 to $12 \mu \mathrm{m}$ wide, well defined waveguides have been achieved. For the inscription of Bragg gratings, the excimer laser beam is shaped into a rectangular geometry using a cylindrical lens arrangement and focused through a phase mask (Ibsen Photonic) in soft contact to the previously written waveguides at a repetition rate of $100 \mathrm{~Hz}$ and a laser fluence of $15 \mathrm{~J} / \mathrm{cm}^{2}$ (measured in front of the phase mask). The applied phase masks feature grating pitches of $\Lambda_{\mathrm{PM}}=1008 \mathrm{~nm}$ for OrmoCore ${ }^{\circledR}$ and $\Lambda_{\mathrm{PM}}=1036.79 \mathrm{~nm}$ for OrmoClad $^{\circledR}$ and OrmoComp $^{\circledR}$. A schematic illustration of the fabrication process and the resulting layer composition is shown in figure 1.

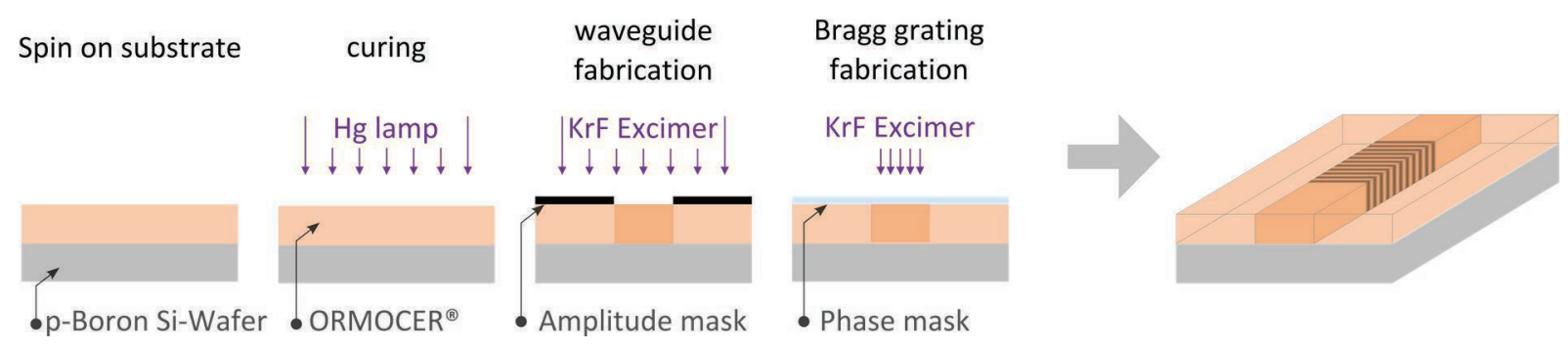

Figure 1: Schematic illustration of the fabrication process of the planar channel waveguides and inscription of the planar Bragg grating.

The fabricated planar sensor chips are bonded to a single mode fiber via butt coupling using a v-groove fiber assembly and are connected to an interrogation system operating at telecom wavelength. The used interrogation system (sm125500 by Micron Optics) operates at a wavelength range between $1510 \mathrm{~nm}$ and $1590 \mathrm{~nm}$ and features a $2 \mathrm{~Hz}$ sampling rate with a resolution of $1 \mathrm{pm}$. The interrogation system furthermore has been expanded by an automatic LabVIEW based Bragg peak detection and tracking which allows to monitor the temporal Bragg wavelength shift. For measuring the Bragg peak response due to temperature changes, the fabricated sensor chips are placed onto a hotplate. A fiber thermometer and a hygrometer are positioned directly on top of the sensor surface serving as a reference for the actual local temperature and relative humidity, respectively. During the measurement, the whole setup is placed in a glove box under constant nitrogen purging in order to guarantee a consistent environment free of external interferences.

\section{Results}

The fabricated ORMOCER ${ }^{\circledR}$ channel waveguide Bragg gratings exhibit a well-defined Bragg reflection with a small FWHM of up to 130 pm and a reflectivity of up to $78 \%$. Figure 2 depicts the reflected spectrum of a fabricated sensor chip, whereas the inset shows the Bragg peak shift due to an increasing temperature. By constituting the relative Bragg wavelength shift with respect to the applied temperature, a linear relationship can be found for all ORMOCER ${ }^{\circledR}$ channel waveguide Bragg grating sensors as depicted in figure 3 . 


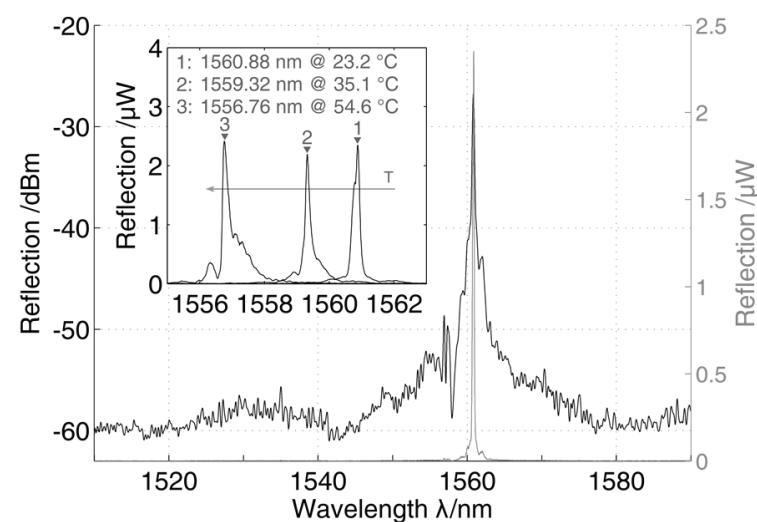

Figure 2: Spectral shift of the OrmoComp ${ }^{\circledR}$ waveguide $B G$ reflection due to temperature changes
The sensors exhibit a sensitivity of $-131 \mathrm{pm} \mathrm{K}^{-1}$ for the OrmoComp ${ }^{\circledR}$, $-238 \mathrm{pm} \mathrm{K}^{-1}$ for the OrmoCore $^{\circledR}$ and -294 pm K$^{-1}$ for the OrmoClad $^{\circledR}$ waveguide Bragg grating. Taking into account the interrogation system's spectral resolution of $1 \mathrm{pm}$, acquisition steps of up to $3.4 \mathrm{mK}$ are possible. However, due to background noises of the interrogation system a Bragg wavelength shift which can be considered as reliable is given for $\Delta \lambda_{B} \geq 3 \sigma$, where the standard deviation of the interrogation system's background noise $\sigma$ was found to be $2 \mathrm{pm}$. Based on these considerations, a minimal detectable temperature shift of $46 \mathrm{mK}$ for the OrmoComp ${ }^{\circledR}, 25 \mathrm{mK}$ for the OrmoCore $^{\circledR}$ and $20 \mathrm{mK}$ for the OrmoClad ${ }^{\circledR}$ channel waveguide Bragg grating can be deduced.

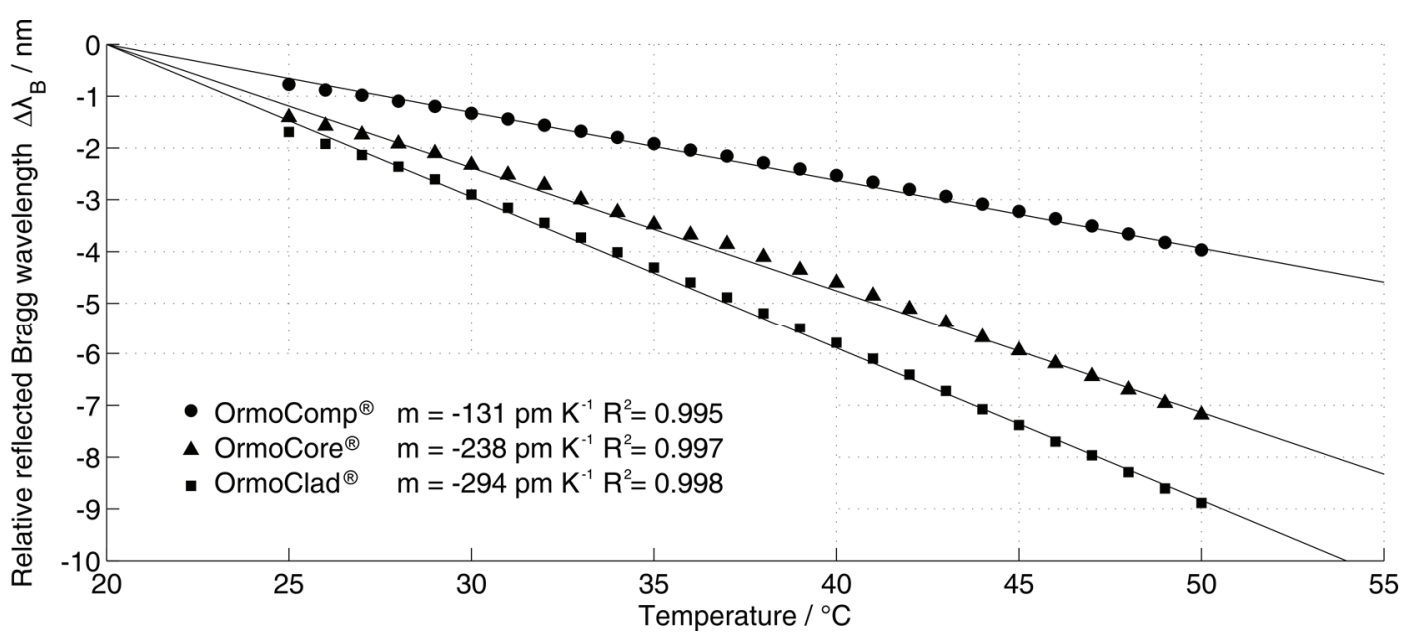

Figure 3: Spectral shift of the channel waveguide Bragg grating reflection due to temperature changes.

The results firstly show the general possibility of producing volumetric channel waveguide Bragg gratings directly into pre-cured ORMOCER ${ }^{\circledR}$, whereas the fabrication method itself is considered fast, cost efficient and reproducible. Secondly, the so produced channel waveguide Bragg grating sensors exhibit a linear dependency of the sensor signal on temperature changes with a withal very high sensitivity which was found to be up to thirty times higher than the sensitivity of common fiber based Bragg grating temperature sensors $[1,2]$. Considering these results, the here described fabrication of waveguide Bragg gratings in ORMOCER ${ }^{\circledR}$ S opens a novel and low-cost possibility to produce highly sensitive and fit for market temperature sensors.

\section{References}

[1] K. O. Hill et al., J. Lightwave Technol.15(8), 1263-1276 (1997); doi:10.1109/50.618320

[2] C. Zhang et al., Electron. Lett. 46(9), 643-644 (2010); doi:10.1049/el.2010.0879

[3] M. Rosenberger et al., Sens. Actuators, A 221, 148-153 (2015); doi: 10.1016/j.sna.2014.10.040
[4] A. Stefani et al. in: Proc. of $35^{\text {th }}$ Optical Fibre Technology (ACOFT), 1-4 (2010); doi: 10.1109/ACOFT.2010.5929947

[5] Z. F. Zhang et al., IEEE Photon. Technol. Lett. 25(3), 310-312 (2013); doi: 10.1109/LPT.2012.2235421

[6] K. Haas and H Wolter, Curr. Opin. Solid State Mater. Sci. 4(6), 471-580 (1999); doi: 10.1016/S1359-0286(00)00009-7

[7] R. Houbertz et al. Thin Solid Films, 442(1), 194200 (2003); doi:10.1016/S0040-6090(03)00982-9

[8] micro resist technology, Processing Guidelines OrmoCore and OrmoClad 2012-11-23 (2012)

[9] micro resist technology, Processing Guidelines OrmoComp 2012-11-5 (2012)

[10] T. Habisreuther et al., IEEE Sensors J. 12(1), 13 16 (2012); doi:10.1109/JSEN.2011.2108280

[11] B. J. Eggleton et al., J. Lightwave Technol. 18(8), 1084-1100 (2000); doi:10.1109/50.857754

[12] R. RajiniKumar et al., IEEE Trans. Appl. Supercond. 16(2), 1737-1740 (2006); doi:10.1109/TASC.2005.864332 